\title{
Gastroenterología y Nutrición como una sola Especialidad de la Pediatría
}

\author{
PAUL R. HARRIS ${ }^{1}$, MARÍA ISABEL HODGSON B. ${ }^{1}$, \\ HUMBERTO SORIANO B. ${ }^{1}$, JUAN CRISTÓBAL GANA A. ${ }^{1}$ \\ 1. Unidad de Gastroenterología y Nutrición Pediátrica, División de Pediatría, Facultad de Medicina, \\ Pontificia Universidad Católica de Chile, Santiago, Chile.
}

La alta complejidad que suelen alcanzar las enfermedades en pediatría ha estimulado el desarrollo de especialidades órgano- o sistemaespecíficas para su mejor estudio, diagnóstico y terapéutica. En ese contexto tanto la gastroenterología como la nutrición en pediatría han crecido con historias paralelas y muchas veces coincidentes.

Las enfermedades del tracto gastrointestinal, del hígado y de la nutrición en el niño chileno son de gran relevancia. En 2006, por ejemplo, la tasa de malnutrición por déficit fue de $3 \%$ en niños menores de 6 años, mientras que la tasa de obesidad alcanzó el $7 \%$ y la de sobrepeso el $15 \%$, en el mismo grupo etáreo (Depto. Estadística e Información de Salud, DEIS, www.minsal.cl). Por otro lado, en niños de $1^{\circ}$ básico, la prevalencia de obesidad alcanzó el 19,4\% (JUNAEB 2006). Las enfermedades del hígado también tienen una alta incidencia. En Chile, en el año 2006 se reportaron más de 13000 casos de hepatitis, la mayoría de ellos en niños y adolescentes. La incidencia de enfermedad hepática severa en el mundo es de aproximadamente dos por 10000 nacidos vivos $^{1}$. En Chile, con 240000 nacidos vivos, es posible estimar una necesidad de 32 a 48 trasplantes pediátricos al año basado en datos internacionales (www.unos.org 2010). Si consideramos una sobrevida actual del transplante hepático del $90 \%$, se podría estimar una preva- lencia futura superior a 800 pacientes trasplantados menores de 18 años de edad. Sin embargo, la cifra de trasplantes pediátricos en Chile era de sólo 8 en 1996 y no ha experimentado el aumento esperado pues en 2007 fue sólo de 9 (Corporación del Trasplante). Los datos de morbilidad en enfermedades del tubo digestivo también son notables. En el año 2005, los egresos hospitalarios por enfermedades del sistema digestivo en niños y adolescentes en Chile fueron cerca de 42000 (8 742 de 0 a 4 años, 10509 de 5 a 9 años y 22743 de 10 a 19 años, reportados por DEIS). Los números mencionados enfatizan la importancia en Chile de enfermedades relacionadas con la Gastroenterología y la Nutrición Pediátrica.

En los países desarrollados de Europa y EE.UU., la Gastroenterología y Nutrición Pediátrica ha alcanzado un alto nivel de desarrollo como especialidad médica. La Sociedad Norteamericana de Gastroenterología, Hepatología y Nutrición Pediátrica se fundó en 1972 y actualmente cuenta con más de 1300 miembros (www.naspghan.org). La Sociedad Europea de la especialidad fue fundada en 1968 y cuenta con más de 600 miembros entre gastroenterólogos, nutriólogos, hepatólogos y científicos que trabajan en esta área (www. espghan.med.up.pt). La formación de postítulo en la especialidad está firmemente establecida y hay más de 50 programas de postítulo

Correspondencia a:

Dr. Paul R. Harris

E-mail: pharris@med.puc.cl 
en EE.UU. que incluyen las universidades más prestigiosas de ese país. El entrenamiento dura tres años y requiere de formación previa en Pediatría por tres años. Los exámenes de Gastroenterología, Hepatología y Nutrición Pediátrica se ofrecen desde hace más de 17 años a graduados de programas acreditados y son, en general, requisito para la práctica de esta especialidad en EE.UU. La revista científica oficial de las sociedades de Gastroenterología y $\mathrm{Nu}$ trición Pediátrica Norteamericana y Europea es el "Journal of Pediatric Gastroenterology and Nutrition" (www.jpgn.org) que cuenta con oficinas editoriales en ambos continentes. Su factor de impacto es 2,07 y su rango es $\mathrm{N}^{\circ} 20$ entre 46 revistas indexadas en el área de Gastroenterología y Hepatología. Los especialistas de Gastroenterología y Nutrición Pediátrica están firmemente conectados a nivel mundial. El 2008, se realizó la $3^{\text {a }}$ reunión mundial de la especialidad organizada por la "Federation of the International Societies for Pediatric Gastroenterology, Hepatology and Nutrition (FISPGHAN)", que agrupa a las sociedades Europea, Norteamericana, Latinoamericana y Asiática de la especialidad (www.fispghan. org). En adición al creciente número de textos de estudio de la especialidad, los gastroenterólogos y nutriólogos de FISPGHAN están conectados por una lista de correo electrónico (pedgi@list.uvm.edu) en la que están inscritos 1726 miembros de todo el mundo, para enviar consultas y comentarios clínicos y académicos. Cabe notar que, aún cuando existen médicos chilenos en otras instituciones que son líderes en la sociedad latinoamericana, no existe por el momento una Sociedad de Gastroenterología, Hepatología y Nutrición en Chile, ni una rama conjunta dentro de la Sociedad Chilena de Pediatría.

En Chile, las especialidades Pediátricas de Gastroenterología y Nutrición se desarrollaron inicialmente en forma separada por razones históricas y de morbilidad prevalente, en gran parte debido a la alta morbi-mortalidad relacionada con la desnutrición infantil en nuestro país en el siglo pasado. La razón del desfase de Chile en la fusión de ambas especialidades probablemente tiene raíces históricas, entre otros factores.
La historia de la nutrición en nuestro país comienza el siglo pasado con el trabajo de pioneros quienes crearon Centros Primarios de Alimentación y/o Nutrición ${ }^{2}$ y destacadas instituciones dedicadas al estudio y fomento de la nutrición como el INTA, el CONIN-CREDES y el CONPAN. En el momento actual, sobre 250 profesionales son miembros permanentes de los 3-4 centros académicos relacionados con problemas de alimentación y nutrición de adultos y niños, trabajando en investigación, enseñanza o extensión: dos de los Centros son de la Universidad de Chile y el otro de la Pontificia Universidad Católica de Chile. Los tres tienen programas de post-grado en nutrición y al menos uno de ellos (INTA) cuenta con un postgrado en planificación alimentaria nutricional (PAN). Existe además un doctorado en nutrición y alimentación, coordinado por la Universidad de Chile. La Sociedad Chilena de Nutrición Bromatología y Toxicología fue fundada en 1943 y en su directorio participan médicos pediatras. Sus propósitos y estatutos, sin embargo (www.sochinut.cl), no mencionan las palabras 'niño', 'pediatría' o 'infantil'. La Sociedad Chilena de Pediatría, por otro lado, cuenta con una Rama de Nutrición fundada hace 25 años. A fines de la década de los ' 80 parte el desarrollo de técnicas modernas de apoyo nutricional intensivo: la nutrición enteral, con sus fórmulas especiales y la nutrición parenteral $^{3}$. La dramática disminución de la desnutrición infantil en Chile en los últimos 50 años es elogio elocuente al trabajo de muchos profesionales dedicados a tratar y prevenir estas enfermedades.

La historia de la gastroenterología pediátrica en Chile se remonta a los años 60, cuando la diarrea crónica asociada a la desnutrición y sus complicaciones eran los problemas principales en la práctica clínica. La mayoría de estos pacientes presentaban infecciones entéricas como la Giardia lamblia. Los primeros especialistas en Chile en los años 70 fueron capaces de reconocer patologías tradicionalmente poco diagnosticadas en Chile como la Enfermedad Celíaca, la Fibrosis Quística del Páncreas y la Enfermedad Inflamatoria Intestinal. En 1969, se funda la Rama de Gastroenterología de la Sociedad Chilena de Pediatría que ha crecido 
y cuenta con más de 43 miembros, la mayoría acreditados como especialistas. Con el auge económico de los últimos 30 años en Chile, y la baja en la tasa de mortalidad infantil en un $82 \%$ desde 1980 , las enfermedades no infecciosas, que constituían un $54 \%$ de las muertes en 1970 subieron a un $75 \%$ en $1991^{4}$. Un resultado de estos cambios epidemiológicos es que patologías como la Enfermedad Inflamatoria Intestinal han aumentado su incidencia. El transplante hepático pediátrico es ya practicado en varios centros en Chile con buenos resultados. La fibrosis quística ya no es una enfermedad rara. El trabajo en niños hospitalizados incluye sobrevivientes de enfermedades graves perinatales como enterocolitis necrotizante, enfermedad de Hirschsprung, parálisis cerebral y síndromes genéticos. El apoyo nutricional al paciente críticamente enfermo o con enfermedades crónicas graves es cada vez más especializado.

El desafío actual, por tanto, es formar médicos en la especialidad de Gastroenterología y Nutrición Pediátrica que estén preparados para tratar con eficiencia estas nuevas y más complejas patologías. Recientemente aparecen signos iniciales en nuestro país a seguir el patrón internacional en actividades educativas, científicas y de extensión conjuntas. Por ejemplo, el año 2007 se realizaron dos cursos de Gastroenterología y Nutrición Infantil con invitados nacionales e internacionales, patrocinados por la Sociedad Chilena de Pediatría. El 2009, Chile fue sede del XVII Congreso Latinoamericano, VIII Congreso Iberoamericano, I Congreso Chileno de Gastroenterología, Hepatología y Nutrición Pediátrica, reflejando la incipiente fusión de ambas especialidades en nuestro país.

Actualmente en Chile hay dos programas de formación en Gastroenterología Pediátrica. El primer programa comenzó hace aproximadamente 10 años, patrocinado por la Universidad de Chile, con un consorcio de hospitales. Recientemente, la Pontificia Universidad Católica de Chile inició un programa de beca de Gastroenterología y Nutrición que cuenta con la participación además de los Hospitales Dr. Sótero de Río y Josefina Martínez. Ambos programas duran dos años y tienen dos cupos al año. Incluye pasantías en los diferentes hospitales, entrenamiento práctico en endoscopias y otros procedimientos de apoyo. Sin embargo, es posible que para lograr un nivel de competencia adecuado en Gastroenterología, Nutrición y Hepatología se requiera de un programa de tres años de duración, como existe en varios centros internacionales, o bien que exista una diferenciación preferencial a una de la disciplinas dentro del $2^{\circ}$ año del programa.

Existen programas de magíster en nutrición en Chile pero con una mención pediátrica y clínica parcial. El programa de Magíster en Nutrición en la PUC se creó el año 1995 y forma de cinco a seis médicos por año, la mayoría con mención en adultos, con dos años de duración. CONACEM no reconoce la Nutrición como especialidad. Existen también programas de nutrición como sub-especialidad de la Medicina Interna. En la Universidad de Chile, existe un programa de Nutrición Pediátrica que es una formación clínica y teórica en el tema para médicos pediatras y generales. Dicho programa no incluye formación en gastroenterología. Recientemente, se está formando un programa de entrenamiento en Nutrición Pediátrica en la Universidad de Chile.

El trabajo en equipo ha permitido la reciente creación en la Universidad Católica de la Unidad de Gastroenterología y Nutrición y el comienzo de un Programa de Postítulo. Una reciente publicación en adultos habla de la tendencia a seguir el modelo pediátrico y al 'matrimonio' entre estas dos especialidades 5 , ya que esta fusión entre Gastroenterología y Nutrición han sido un aporte invaluable para ofrecer un completo manejo multidisciplinario. Sin perjuicio de que existan 'super-especialistas', esperamos que la tendencia a unir la Gastroenterología, Hepatología y Nutrición en Pediatría (independiente del nombre que adquiera) siga en Chile, camino que creemos beneficia el cuidado integral de los niños en esta área, optimiza la formación de estudiantes y potencia las áreas de investigación.

Es evidente que la formación de unidades conjuntas no pretende que los profesionales desarrollen competencias similares, dado que la formación natural lleva a la subespecialización en hígado, páncreas, nutrición enteral 
o alergia alimentaria, por ejemplo. Muy por el contrario, la creación de unidades conjuntas pretende que cada médico (y a futuro cada educando) esté expuesto a un núcleo central formativo que enriquezca su formación para luego avanzar en el camino profesional. Las limitantes de este modelo (dificultades en la profundización en algunas áreas, la falta de competencia en procedimientos complejos, etc) serán superados en la medida que las futuras unidades creen redes flexibles en la formación, con espacio para el desarrollo individual.

\section{Referencias}

1.- Arya $G$, Balistreri WF: Pediatric liver disease in the United States: epidemiology and impact. J Gastroenterol
Hepatol 2002; 17: 521-5.

2.- Mönckeberg F: Prevención de la desnutrición en Chile experiencia vivida por un actor y espectador. Rev Chil Nutr 2003; 30: S1.

3.- Hodgson MI, Rathkamp B, Brunet M, Uauy R, Torres $J$ : Alimentación de recién nacido de muy bajo peso con leche de su propia madre. Rev Chil Pediatr 1987; 58: 296-301.

4.- Guiraldes E: Pediatric Gastroenterology in Chile: a personal perspective. J Pediatr Gastroenterol Nutr 2002; 33: $231-2$.

5.- Report of the Multisociety Task Force on GI Training. Gastroenterol 2009; 137: 1839-1843. http://www. gastrojournal.org/article/S0016-5085(09)01155-X/ preview-article-footnote-1\#article-footnote-1 http:// www.gastrojournal.org/article/S0016-5085(09)01155$\mathrm{X} /$ preview- article-footnote-2\#article-footnote-2.

\section{A LOS AUTORES}

Comunicamos a los autores de los artículos presentados para publicación en Revista Chilena de Pediatría, que ha concluido exitosamente el Proyecto Editorial del Fondo de Publicación de Revistas Científicas CONICYT 2008-2009 "Revista Chilena de Pediatría: De las Publicaciones en Papel a un Proceso Editorial On-Line basado en Open Journal System". Por lo tanto, solicitamos a los autores de los artículos presentados a partir de ahora, que ingresen a www.revistachilenadepediatria.cl, se registren e identifiquen de acuerdo a las instrucciones, y procedan a ingresar su contribución al sistema editorial electrónico según se detalla.

La Sociedad Chilena de Pediatria y Revista Chilena de Pediatría se enorgullecen de poner a disposición de nuestros colaboradores esta moderna herramienta editorial, en forma pionera entre las publicaciones biomédicas de nuestro país.

El Editor 